\title{
Systematic analysis of NAC transcription factors in Gossypium barbadense uncovers their roles in response to Verticillium wilt
}

\author{
Zhanji Liu ${ }^{\text {Corresp., }}{ }^{1}$, Mingchuan Fu ${ }^{1}$, Hao Li ${ }^{1}$, Yizhen Chen ${ }^{1}$, Liguo Wang ${ }^{1}$, Renzhong Liu ${ }^{1}$ \\ ${ }^{1}$ Key Laboratory of Cotton Breeding and Cultivation in Huang-Huai-Hai Plain, Ministry of Agriculture, Cotton Research Center of Shandong Academy of \\ Agricultural Sciences, Jinan, China \\ Corresponding Author: Zhanji Liu \\ Email address: scrcliuzhanji@sina.com
}

As one of the largest plant-specific gene families, the NAC transcription factor gene family plays important roles in various plant physiological processes that are related to plant development, hormone signaling, and biotic and abiotic stresses. However, systematic investigation of the NAC gene family in sea-island cotton (Gossypium babardense L.) has not been reported, to date. The recent release of the complete genome sequence of seaisland cotton allowed us to perform systematic analyses of G. babardense NAC (GbNAC) genes. In this study, we performed a genome-wide survey and identified 270 GbNAC genes in the sea-island cotton genome. Genome mapping analysis showed that GbNAC genes were unevenly distributed on 26 chromosomes. Through phylogenetic analyses of GbNACs along with their Arabidopsis counterparts, these proteins were divided into 10 groups $(I-X)$, and each contained a different number of GbNACs with a similar gene structure and conserved motifs. One hundred and fifty-four duplicated gene pairs were identified, and almost all of them exhibited strong purifying selection during evolution. In addition, various cis-acting regulatory elements in GbNAC genes were found to be related to major hormones, defense and stress responses. Notably, transcriptome data analyses unveiled the expression profiles of 62 GbNAC genes under Verticillium wilt (VW) stress.

Furthermore, the expression profiles of 15 GbNAC genes tested by quantitative real-time PCR (qPCR) demonstrated that they were sensitive to methyl jasmonate (MejA) and salicylic acid (SA) treatments and that they could be involved in pathogen-related hormone regulation. Taken together, the genome-wide identification and expression profiling pave new avenues for systematic functional analysis of GbNAC candidates, which may be useful for improving cotton defense against VW. 


\section{Systematic analysis of NAC transcription factors in}

\section{Gossypium barbadense uncovers their roles in}

\section{3 response to Verticillium wilt}

4 Zhanji Liu ${ }^{1 *}$, Mingchuan $\mathrm{Fu}^{1}$, Hao Li ${ }^{1}$, Yizhen $\mathrm{Chen}^{1}$, Liguo Wang ${ }^{1}$, Renzhong Liu ${ }^{1}$

$5{ }^{1}$ Key Laboratory of Cotton Breeding and Cultivation in Huang-Huai-Hai Plain, Ministry of

6 Agriculture, Cotton Research Center of Shandong Academy of Agricultural Sciences, Jinan

7250100, China

8 *Corresponding Author:

9 Zhanji Liu

10202 Gongyebei Road, Jinan, Shandong, 250100, China

11 E-mail address: scrcliuzhanji@sina.com 


\section{ABSTRACT}

As one of the largest plant-specific gene families, the NAC transcription factor gene family plays important roles in various plant physiological processes that are related to plant development, hormone signaling, and biotic and abiotic stresses. However, systematic investigation of the NAC gene family in sea-island cotton (Gossypium babardense L.) has not been reported, to date. The recent release of the complete genome sequence of sea-island cotton allowed us to perform systematic analyses of G. babardense NAC (GbNAC) genes. In this study, we performed a genome-wide survey and identified 270 GbNAC genes in the sea-island cotton genome. Genome mapping analysis showed that $G b N A C$ genes were unevenly distributed on 26 chromosomes. Through phylogenetic analyses of GbNACs along with their Arabidopsis counterparts, these proteins were divided into 10 groups (I-X), and each contained a different number of GbNACs with a similar gene structure and conserved motifs. One hundred and fifty-four duplicated gene pairs were identified, and almost all of them exhibited strong purifying selection during evolution. In addition, various cis-acting regulatory elements in $G b N A C$ genes were found to be related to major hormones, defense and stress responses. Notably, transcriptome data analyses unveiled the expression profiles of 62 GbNAC genes under Verticillium wilt (VW) stress. Furthermore, the expression profiles of 15 GbNAC genes tested by quantitative real-time PCR (qPCR) demonstrated that they were sensitive to methyl jasmonate (MeJA) and salicylic acid (SA) treatments and that they could be involved in pathogen-related hormone regulation. Taken together, the genome-wide identification and expression profiling pave new avenues for systematic functional analysis of GbNAC candidates, which may be useful for improving cotton defense against VW.

Keywords: sea-island cotton; NAC transcription factor; genome-wide survey; Verticillium wilt 


\section{INTRODUCTION}

The plant-specific $N A C$ genes ( $\underline{N A M}$, no apical meristem; $\underline{A} T A F$, Arabidopsis transcription activation factor; and $\underline{C} U C$, cup-shaped cotyledon) form one of the largest families of transcription factors (Nuruzzaman et al., 2013). Typically, NAC proteins harbor a highly conserved NAC domain at the $\mathrm{N}$-terminal and a variable transcriptional regulatory region (TR) at the $\mathrm{C}$-terminal. The NAC domain can be further divided into five subdomains (A-E) and functions as DNA binding, nuclear localization, and formation of homodimers or heterodimers, while the TR region is responsible for transcription regulation as either an activator or a repressor (Olsen et al., 2005). $N A M$, the first $N A C$ gene, was discovered in Petunia and functions in determining positions of shoot apical meristems and primordia (Souer et al., 1996). Since then, a large number of NAC genes have been identified from diverse plant species (Nuruzzaman et al., 2010; Liu et al., 2019).

Accumulated evidences indicate that the functions of $N A C$ genes are associated with almost every biological process in plants, such as leaf senescence (Wang et al., 2015; Zhao et al., 2016), secondary cell wall formation (Zhang et al., 2018), and hormone signaling (Takasaki et al., 2015). Notably, a number of NAC genes, especially ATAF subfamily members, were proven to serve as critical regulators in plant defense against biotic and abiotic stresses (Nuruzzaman et al., 2013; Karanja et al., 2017). In Arabidopsis, ANAC032 was induced by bacterial pathogen Pst (Pseudomonas syringae pv. tomato DC3000) infection, and SA and jasmonic acid (JA) treatments. Furthermore, transgenic Arabidopsis plants overexpressing ANAC032 showed strongly enhanced resistance to Pst, while the ANAC032 knockout mutant exhibited increased susceptibility to Pst (Allu et al., 2016). In rice, the expressions of $S N A C 1$ were enhanced under drought, salt, and cold stresses. Overexpression $S N A C l$ in rice resulted in increased tolerance to drought (Hu et al., 2006). Similarly, transgenic $O S N A C 111$ showed improved resistance to blast fungus in rice by regulating the expression of several defense-related genes (Yokotani et al., 2014).

Cotton is the most important natural fiber crop, and is also used as a food crop due to high levels 
of vegetable oil and protein in cottonseeds ( $\mathrm{Li}$ et al., 2009). However, cotton production can be dramatically decreased by the occurrence of VW, a devastating vascular disease caused by Verticillium dahliae (Sun et al., 2013). Typically, infected plants show leaf chlorosis, leaf shedding, vascular discoloration, wilting, and plant death. In general, upland cotton (Gossypium hirsutum), accounting for about $90 \%$ of annual world cotton production, is susceptible to VW, whereas sea-island cotton, accounting for approximately $5 \%$ of annual world cotton output, is immune to VW. Thus, extensive efforts have been made to investigate the molecular mechanism of sea-island cotton resistance to VW (Zhang et al., 2015). Recently, a NAC gene GbNAC1 was identified from $G$. barbadense. Overexpression of $G b N A C 1$ in Arabidopsis can significantly enhance resistance to $\mathrm{VW}$, implying that G. barbadense $N A C$ genes might play pivotal roles in biotic stress resistance (Wang et al., 2016). The availability of diploid and tetraploid cotton genomes has allowed scientists to identify the $N A C$ gene family members at the genome-wide scale, such as 145 genes in G. raimondii (Shang et al., 2013), 141 in G. arboreum (Shang et al., 2016; Fan et al., 2018), and 283 in G. hirsutum (Sun et al., 2018). However, systematic analysis of $N A C$ genes in $G$. babardense has not been completed. G. babardense $\left(\mathrm{AD}_{2}\right)$ and $G$. hirsutum $\left(\mathrm{AD}_{1}\right)$ are allotetraploids, which evolve from transoceanic hybridization between an A-genome species immigrated from Africa and a native American D-genome species, while G. arboreum $\left(\mathrm{A}_{2}\right)$ and G. raimondii $\left(\mathrm{D}_{5}\right)$ are diploids resembling the A-genome and D-genome progenitor, respectively (Hu et al., 2019). In this study, we performed a genome-wide survey and identified 270 GbNAC genes in the latest G. babardense genome released by Wang et al. (2019). These GbNAC genes were classified into 10 groups on the basis of sequence similarity. Sequence comparison among $G b N A C$ genes revealed the presence and distribution of duplicated genes. Additionally, to identify $G b N A C$ candidate genes associated with VW resistance, we analyzed the expression patterns of $G b N A C$ genes using available transcriptome data of G. babardense cv. 7124 inoculated with the fungal pathogen $V$. dahliae. Subsequently, qPCR-based gene expression profiling demonstrated that selected $G b N A C$ candidate genes might be involved in MeJA and SA regulation. This study provides comprehensive information about sea-island cotton $N A C$ genes, as 
100

101

102

103

104

105

106

107

108

109

110

111

112

113

114

115

116

117

118

119

120

121

122

123

124

well as a foundation for in-depth functional analysis of novel $G b N A C$ candidate genes, which may be useful for the improvement of pathogen resistance in cotton.

\section{MATERIALS AND METHODS}

\section{Identification of $N A C$ genes in the sea-island cotton genome}

We downloaded the genome sequences (v. HAU) of sea-island cotton from the CottonGen database (https://www.cottongen.org/, Wang et al., 2019). The Hidden Markov Model (HMM) profile of the NAC domain (PF02365) was retrieved from the Pfam database (http://pfam.xfam.org/, Finn et al., 2016). The HMMER program was used to search NAC protein in the sea-island cotton genome (Finn et al., 2011) with an E-value cutoff of $\mathrm{e}^{-5}$. Then, all the putative proteins were confirmed by the Pfam and SMART database (http://smart.emblheidelberg.de/, Letunic and Bork, 2018). The MW (molecular weight) and pI (theoretical isoelectric point) of each NAC protein were predicted by the online software ExPASy (https://www.expasy.org/, Artimo et al., 2012). The TMHHM server (v. 2.0, http://www.cbs.dtu.dk/services/TMHMM/) was used to identify membrane-bound NAC proteins (Krogh et al., 2001). CELLO (v. 2.5, subCELlular Localization predictor, http://cello.life.nctu.edu.tw/, Yu et al., 2006) was used to predict the subcellular localization of GbNACs.

\section{Multiple alignments, phylogenetic analysis, gene duplication and synteny analysis}

Multiple sequence alignments were performed with the NAC domain sequences of the GbNAC proteins using MEGA X (https://www.megasoftware.net/, Kumar et al., 2018). A phylogenetic tree was constructed by the neighbor-joining method with the following parameters: Poisson correction, pairwise deletion, and 1000 bootstrap replicates. Gene duplications were analyzed with two major criteria, that is, the length of the aligned sequence covers more than $75 \%$ of the longer gene and similarity of the aligned regions is greater than $75 \%$ (Vatansever et al., 2016). Alignment of the coding sequences of duplicated genes was performed by the Clustal X (v. 2.0) program 
125 (Larkin et al., 2007), and the values of nonsynonymous (Ka) and synonymous (Ks) substitution 126 rates were calculated using KaKs_Calculator package (Zhang et al., 2006) via model averaging.

127 The approximate date of duplication events (million years ago, Mya) was estimated using the 128 formula $\mathrm{T}=\mathrm{Ks} / 2 \lambda \times 10^{-6}$, on the basis of molecular clock rate of $2.6 \times 10^{-9}$ 129 substitutions/synonymous site for cotton (Liu et al., 2015). The relationships of duplicated genes 130 were illustrated with the Circos program (Krzywinski et al., 2009). MCScanX was used to detect 131 the synteny of $N A C$ genes between G. barbadense and the other plant species (Wang et al., 2012).

\section{Gene structure, chromosomal mapping, and conserved motif analysis}

133 The gene structures were determined using the CDS and DNA sequences of $G b N A C$ genes and 134 visualized by the Gene Structure Display Server (http://gsds.cbi.pku.edu.cn/, Hu et al., 2015). The 135 positions of these $G b N A C$ genes were determined by using the nucleotide sequence as a query to 136 search against the $G$. barbadense genome. In addition, chromosomal localization map was 137 constructed by using the MapChart (v. 2.32) program (Voorrips 2002). In order to identify the 138 conserved motifs among all the $G b N A C$ genes, their protein sequences were subjected to the online 139 software MEME (http://meme-suite.org/tools/meme, Bailey et al., 2015) using default parameters 140 with exception for number of motif. The number of motifs was set to 20.

141

142

143

144

145

146

147

148

149

150

\section{Cis-acting regulatory element and miRNA target analysis}

For cis-acting regulatory element analysis, we retrieved 1,500 bp DNA sequences up-stream from the transcription start site from the newly released G. barbadense genome sequences (Wang et al., 2019) and then screened them in the PlantCare (http://bioinformatics.psb.ugent.be/webtools/plantcare/html/, Lescot et al., 2002) and PLACE (https://www.dna.affrc.go.jp/PLACE/, Higo et al., 1999) databases. For miRNA target analysis, we downloaded all the mature miRNA sequences of Gossypium from the miRBase database (v. 22.1) (http://www.mirbase.org/). The online sever psRNATarget (http://plantgrn.noble.org/psRNATarget/, Dai et al., 2018) was used to predict the miRNA target. 
151 The transcriptome data of sea-island cotton were obtained from the Sequence Read Archive under 152 accession No. SRP03537 at the NCBI website

153 (http://www.ncbi.nlm.nih.gov/sra/?term=SRP035371, Chen et al., 2015), where the plants were 154 inoculated with $V$. dahliae. Briefly, two-week-old seedlings of $G$. barbadense resistant cultivar 1557124 were inoculated with the high virulence V991 defoliating strain of $V$. dahliae $\left(5 \times 10^{6}\right.$ 156 spores $/ \mathrm{mL}$ ) by the root-dip method for $2,6,12,24,48$, and $72 \mathrm{~h}$. Then, the samples, including the 157 mock-inoculated (control) and six inoculated, were collected for RNA sequencing. We retrieved 158 the expression data of GbNAC genes from the root under $V$. dahliae infection (Chen et al., 2015). 159 The hierarchical clustering and the heatmap-based expression profiles of $G b N A C$ genes were performed using ClustVis (Metsalu and Vilo 2015). The Venn map of differentially expressed GbNAC genes was constructed using the UpSetR package (Lex et al., 2014).

\section{RNA isolation and qPCR analysis}

G. barbadense cv. 7124 seeds were cultivated in commercial soil at $28^{\circ} \mathrm{C}$ with a photoperiod of

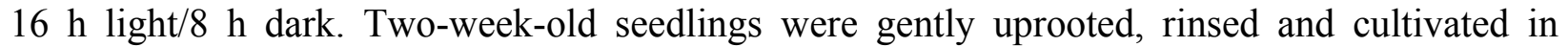
Hoagland solution for two days. Then these seedlings were treated with Hoagland solution containing 0.1 mM MeJA and $1 \mathrm{mM} \mathrm{SA}$, respectively. Roots were sampled from three biological replicates after treatment for $1,2,6$, and $12 \mathrm{~h}$, then immediately immersed in liquid nitrogen and stored at $-80^{\circ} \mathrm{C}$ for $\mathrm{qPCR}$.

The total RNA from the root samples treated with MeJA and SA and the control (roots from Hoagland solution without hormone) was extracted using Trizol (Invitrogen). The first strand cDNA was generated from $1 \mu \mathrm{g}$ of total RNA using a PrimerScript ${ }^{\mathrm{TM}} 1$ st Strand cDNA Synthesis Kit (Takara, Dalian, China). qPCR was performed with three replicates using an ABI QuantStudio 5 Real-Time PCR System (Thermo Fisher Scientific, Waltham, MA, USA) and SYBR ${ }^{\circledR}$ Premix Ex $\operatorname{Taq}^{\mathrm{TM}}$ (Takara, Dalian, China). The amplification procedure was as follows: one cycle at $95^{\circ} \mathrm{C}$ for $3 \mathrm{~min}$; then 40 cycles at $95^{\circ} \mathrm{C}$ for $15 \mathrm{~s}, 60^{\circ} \mathrm{C}$ for $15 \mathrm{~s}$. The cotton actin (AF059484) was selected as the internal reference gene (Zhang et al., 2013). Gene expression levels were calculated 
177 according to the $2^{-\Delta \Delta \mathrm{Ct}}$ method described by Livak \& Schmittgen (2001). The primers used for 178 qPCR are listed in Table S1.

\section{RESULTS}

\section{Identification and phylogenetic analysis of NAC family members in Gossypium barbadense}

A total of 270 NAC proteins were identified from G. barbadense and named as GbNAC001 to GbNAC270. All of the 270 GbNACs contained the NAC domain (PF02365) based on Pfam and SMART tests. The lengths of GbNAC proteins ranged from 154 (GbNAC059) to 959 (GbNAC175) amino acids with MW from 17.65 to $107.75 \mathrm{kDa}$, and pI from 4.67 to 9.79 . Subcellular localization of GbNACs was predicted using the online software CELLO (http://cello.life.nctu.edu.tw/). Among the 270 GbNAC proteins, three were predicted to be mitochondrial proteins (GbNAC031, 032, and 255); five were located in the chloroplast (GbNAC014, 036, 116, 174, and 249); 10 were extracellular; 23 were cytoplasmic, and the rest were localized in the nucleus. These results are similar to those of cucumber (Liu et al., 2018). Detailed information including gene locus, chromosome location, exon number, sequence length, MW, pI, Arabidopsis orthologous locus and subcellular location of all identified GbNAC proteins is provided in Supplementary Table S2.

The NAC domain sequences of GbNAC proteins were used to construct a neighbor-joining phylogenetic tree. As a result, the 270 GbNACs were classified into 10 groups which were named as I to X (Figure 1). Group VI contained the most NAC members with 47 GbNACs, followed by group I with 39 GbNACs. Group III had the least NAC members with only ten GbNACs. Additionally, 35 GbNACs with high similarity to Arabidopsis ATAF members were clustered in group IV. Arabidopsis ATAF members play pivotal roles in the responses to biotic and abiotic stresses. These results suggest that NAC members in group IV may have similar functions with Arabidopsis ATAF members.

\section{Chromosomal locations, duplications, and synteny analysis of the $G b N A C$ genes}


202 To determine the chromosomal distribution of the $G b N A C$ genes, we searched the sea-island cotton 203 genome database using blastn and the DNA sequence of each $G b N A C$ gene. The results suggested 204 that $263 G b N A C$ genes (97.4\%) were mapped to 26 chromosomes. Specifically, 132 GbNAC genes were distributed in the A-subgenome, and 131 were located in the D-subgenome (Figure 2). In

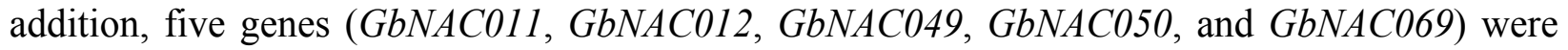
anchored in four A-subgenome scaffolds, and two genes (GbNAC148 and GbNAC262) were found in two D-subgenome scaffolds. The number of $G b N A C$ genes distributed on each chromosome was uneven. Chromosome D11 contained the highest number of $N A C$ genes, with $15 G b N A C$ genes. In contrast, chromosome A10 contained the least number of $N A C$ genes, with only five genes. Interestingly, many $G b N A C$ genes were clustered within a short distance, such as the top of A11 and D11 and the bottom of A08 and D08.

Gene duplication including tandem duplication, segmental duplication, and whole-genome duplication (WGD) is a major driving force in the evolution of plants. The origin of multigene family is due to gene duplication that arose from region-specific duplication or WGD ( $D u$ et al., 2012). To reveal the expansion mechanism of the $G b N A C$ gene family, gene duplication analysis was performed using blastn and the coding sequences (cds) of all GbNAC genes. In all, we identified 148 pairs (212 GbNAC genes) of segmental duplications, three pairs of tandem duplications (GbNAC011/GbNAC012, GbNAC024/GbNAC025, and GbNAC082/GbNAC083), and one triplicate repeat of tandem duplications (GbNAC212/GbNAC213/GbNAC214) (Figure 3). One hundred and thirty-two duplication gene pairs occur between the A-subgenome and the Dsubgenome, while only 12 and 10 duplication gene pairs occur within the A-subgenome and the D-subgenome, respectively. These genes represent approximately 81.9\% (221 of 270) of the GbNAC genes, indicating their origin may be from sea-island cotton genome duplication events. Using the Ka and Ks of each duplicated $G b N A C$ gene pair, we found that the Ks values of all gene pairs were between 0.008 and 0.912 . Specifically, the Ks values of 87 (58.78\%) gene pairs were less than 0.05 . Additionally, the $\mathrm{Ka} / \mathrm{Ks}$ value of each gene pair was calculated and the $\mathrm{Ka} / \mathrm{Ks}$ values of 140 gene pairs $(94.59 \%)$ were less than 1, which indicated these genes had evolved under strong 
229

230

231

232

233

234

235

236

237

238

239

240

241

242

243

244

245

246

247

248

249

250

251

252

253

254

purifying selection. Furthermore, eight gene pairs $(\mathrm{Ka} / \mathrm{Ks}>1)$ may evolve under strong positive selection after duplication. Moreover, we also calculated the approximate date of duplication events. The duplication events of $G b N A C$ genes occurred from $1.58 \mathrm{Mya}(\mathrm{Ks}=0.008)$ to 175.29 Mya $(\mathrm{Ks}=0.912)$, with a mean of 59.31 Mya $(\mathrm{Ks}=0.154)$. Detailed information including duplication gene pairs, chromosome location, duplication type, $\mathrm{Ka}, \mathrm{Ks}, \mathrm{Ka} / \mathrm{Ks}$ and approximate duplication date (Mya) of all identified duplicated $G b N A C$ genes is provided in Supplementary Table S3.

To detect the synteny of $N A C$ genes, we performed a collinearity analysis between G. barbadense and the other four plant species (Arabidopsis thaliana, G. arboretum, G. raimondii and $G$. hirsutum) using MCScanX. Previously, 117, 141, and 145 NAC genes were revealed in A. thaliana (AtNAC, Nuruzzaman et al., 2010), G. arboretum (GaNAC, Shang et al., 2016), and G. raimondii (GrNAC, Shang et al., 2013), respectively. For $N A C$ genes in G. hirsutum (GhNAC), we identified 272 GhNAC genes in the upland cotton genome that was released recently (Wang et al., 2019). Totally, $945 N A C$ genes were used to evaluate synteny relationship in this study. As a result, we found 421 paired collinearity relationships between $247 G b N A C$ and $234 G h N A C$ genes, 241 pairs between $241 G b N A C$ and 133 GrNAC genes, 181 pairs between 120 GbNAC and $54 A t N A C$ genes, and 142 pairs between 141 GbNAC and 81 GaNAC genes (Figure 4 and Table S4). Notably, 69 GbNAC genes are collinear with NAC genes from the other four species (Table S4).

\section{GbNAC gene structures and conserved motifs}

To better understand the relationship between gene function and evolution among the GbNAC genes, the exon/intron organization and conserved motifs were analyzed (Figure 5). The number of exons ranged from 1 to 10 . Most $G b N A C$ genes $(182 / 270,67.41 \%)$ had three exons (Figure 5), although GhNAC059, GhNAC105, and GhNAC238 contained only one exon, and GhNAC129 and GhNAC175 contained 10 exons, the highest number of all genes. We also found that $G b N A C$ genes in the same group had a similar gene structure. For example, all 12 members in group IX had three exons. Among the 39 members in group I, all had three exons except GbNAC060 and GbNAC145 
255

256

257

258

259

260

261

262

263

264

265

266

267

268

269

270

271

272

273

274

275

276

277

278

279

280

(Figure 5).

Twenty conserved motifs were identified among the 270 GbNAC proteins (Figure S1 and Table S5). As a result, all GbNAC proteins contain a conserved NAC domain at the N-terminal, which includes five subdomains (A-E, Figure 6). Notably, the members with high similarity in the same group shared a common motif composition. For example, GbNAC213 and GbNAC214 were found to contain the same three motifs (Figure 5). This finding indicates that these genes may have similar functions. Most GbNAC proteins (257/270, 95.19\%) contain 2-5 conserved motifs. However, seven GbNAC proteins (GbNAC033, GbNAC068, GbNAC092, GbNAC109, GbNAC200, GbNAC226, and GbNAC242) contain only one motif and six GbNAC proteins (GbNAC017, GbNAC020, GbNAC074, GbNAC160, GbNAC206, and GbNAC253) contain six motifs. Additionally, we found that subdomains A (246/270), and/or C (200/270), and/or D $(250 / 270)$ are present in most GbNAC proteins. Furthermore, we found that $231 \mathrm{GbNAC}$ proteins contained the conserved histidine residue in subdomain D, which influences homodimerization and DNA binding of NAC proteins (Kang et al., 2018).

Membrane-bound NAC proteins feature a distinctive transmembrane motif (TM) at either the C terminal region or the $\mathrm{N}$ terminal region and play vital roles in plant defense against abiotic stresses (Kim et al., 2010; Sun et al., 2018). In the sea-island cotton genome, 22 membrane-bound GbNAC proteins were identified (Table S2). Notably, 20 membrane-bound GbNAC proteins contain one $\mathrm{TM}$ at the $\mathrm{C}$ terminal and two GbNAC members (GbNAC110 and GbNAC243) contain one TM at the $\mathrm{N}$ terminal. These membrane-bound GbNAC proteins are distributed in three groups: seven in group V, 11 in group VII, and four in group VIII.

\section{Cis-acting regulatory element and miRNA target analysis}

To better understand the transcriptional regulation mechanisms of $G b N A C$ genes, we characterized the cis-acting regulatory elements within a $1500 \mathrm{bp}$ upstream region from the transcription start site using PlantCARE and PLACE database (Table S6). A large number of cis-acting regulatory elements were identified in promoter sequences of 270 GbNAC genes (Figure S3 and Table S7). 
281 Common regulatory elements such as TATA-box and CAAT-box were present in all GbNAC

282

283

284

285

286

287

288

289

290

291

292

293

294

295

296

297

298

299

300

301

302

303

304

305

306

307 genes. Meanwhile, we identified 11 cis-acting regulatory elements related to hormone responses. Among these, AuxRR-core and TGA-element, auxin-responsive element; ABRE, MYB, and MYC, cis-element involved in abscisic acid (ABA) responsiveness; GARE, P-box, and TATCbox, involved in gibberellin responsiveness; ERE, ethylene responsive element; CGTCA-motif, MeJA responsive elements; and TCA-element, involved in SA responsiveness. Notably, all of the $270 G b N A C$ genes contained at least one hormone-responsive element (Figure S3). MYB, MYC, and ERE were available in at least $80 \%$ of the $G b N A C$ genes. Additionally, promoter sequences of some GbNAC genes also contained several elements involved in biotic and abiotic stress responses, including pathogen defense (AT-rich and TC-rich repeat), drought (MBS), cold (DRE and LTR), anaerobic stress (ARE), and wounding (WUN-motif). Thus, GbNAC genes could be regulated by diverse hormone and environmental changes.

Recent reports have defined a subset of genes from the NAC-domain gene family as potential targets of miRNAs. To determine the involvement of miRNAs in regulating the expression of GbNAC genes, putative miRNA targets were determined in the 270 GbNAC genes using the online sever psRNATarget with the default parameters except Expection $(\leq 3.0)$. Totally, $43 G b N A C$ genes were predicted as the targets of 21 known miRNAs (Table S8). Seven GbNAC genes were each predicted to be the targets of two miRNAs and GbNAC007 was the target of three miRNAs (gra-miR8634, gra-miR8786a and gra-miR8786b). Specifically, miR164 targets 14 GbNAC genes, which are all from group II (Table S8). In Arabidopsis, the miR164 family (ath-miR164a/b/c) guides the cleavage of the transcripts of five NAC genes (NAC1/At1G56010, CUC1/At3g15170, CUC2/At5g53950, ANAC080/At5g07680, and ANAC100/At5g61430) that function in the regulation of plant growth and development such as lateral root emergence, formation of vegetative and floral organs, and age-dependent cell death (Fang et al., 2014; Hernandez \& SananMishra 2017). The 14 miR164-targeted $G b N A C$ genes and the five Arabidopsis $N A C$ genes were clustered into group II, indicating that these genes have high sequence identity and may have a similar function. 
308

309

310

311

312

313

314

315

316

317

318

319

320

321

322

323

324

325

326

327

328

329

330

331

332

333

334

\section{Expression profile of $G b N A C$ genes in response to Verticillium wilt and hormones}

We used publicly available transcriptome data to assess expression of $G b N A C$ genes in roots under VW stress. As a result, 239 (88.52\%) GbNAC genes were identified to be expressed in infected root samples (Figure 7). Three patterns (Pattern I-III) of expression were revealed. Genes from Pattern I have low expression levels in the control and then were up-regulated gradually during VW infection. Genes from Pattern II, in contrast to Pattern I, were highly expressed in the control and then were subsequently down-regulated during VW infection. Genes from Pattern III showed high expression levels in the control, then were down-regulated at the early stages $(2,6$ and $12 \mathrm{~h})$ of inoculation, and finally up-regulated at the late stages $(24,48$ and $72 \mathrm{~h})$ of inoculation. There are 130 duplicated gene pairs among the 239 GbNAC genes. Most duplicated gene pairs (80.77\%) demonstrated similar expression patterns, suggesting that duplicated genes are functionally redundant. However, some duplicated genes have a divergent expression. For example, GbNAC082 and GbNAC083 are tandem duplication genes. GbNAC082 had considerably high expression in the control and was up-regulated by VW stress at $2 \mathrm{~h}$, while GbNAC083 had low expression in the control and was down-regulated remarkably by VW stress at $2 \mathrm{~h}$.

The expression of $192 G b N A C$ genes was significantly altered $\left(\left|\log _{2}{ }^{\text {Fold }}\right| \geq 1\right)$ in roots inoculated with $V$. dahliae as compared to the control in at least one time point (Figure S2). 103, 130, 123, 135, 130, and $128 G b N A C$ genes were significantly induced at 2, 6, 12, 24, 48, and $72 \mathrm{~h}$ after inoculation, respectively, which indicates the number of differentially expressed $G b N A C$ genes was similar among different time points. Additionally, the expression of $62 G b N A C$ genes including 15 up-regulated and 47 down-regulated genes were significantly altered at all of the six time points (Figure S2 and Table S9). Eighteen duplicated gene pairs were revealed among the 62 GbNAC genes. Notably, GbNAC genes in each duplicated gene pair had similar expression patterns. The expression of previously reported sea-island cotton $N A C$ gene GbNAC1 (GbNAC220 in this study) was also altered. $G b N A C 1$, a positive regulator involved in cotton resistance to $V$. dahliae, was down-regulated in infected roots compared to the control, which is consistent with qPCR results reported by Wang et al. (2016). These results imply that GbNAC genes could play 
335

336

337

338

339

340

341

342

343

344

345

346

347

348

349

350

351

352

353

354

355

356

357

358

359

crucial roles in the defense of cotton against VW.

Phytohormones, such as MeJA and SA, regulate plant defenses against diverse pathogens. In order to identify hormone-responsive GbNAC genes, G. barbadense cv. 7124 seedlings were treated with MeJA and SA, and the changes in transcript abundance of 15 genes selected from the 62 differentially expressed $G b N A C$ genes were analyzed by qPCR. As shown in Figure 8, all the GbNAC genes tested were sensitive to the hormones MeJA and SA, but the levels of sensitivity were substantially different at the four time points. In general, most tested $G b N A C$ genes were upregulated under MeJA treatment, but down-regulated under SA treatment. Specifically, the expression of two $G b N A C$ genes ( $G b N A C 014$ and $G b N A C 164$ ) from group IV were up-regulated at all the four time points, and had at least 186 -fold and 225 -fold increase at $6 \mathrm{~h}$ compared with control, respectively (Figure 8).

\section{DISCUSSION}

\section{Characterization of $G b N A C$ genes}

In this study, we performed a genome-wide analysis of the sea-island cotton $G b N A C$ gene family to investigate their potential functions in response to VW. As a result, $270 \mathrm{GbNAC}$ genes were revealed in the G. barbadense genome, which is similar to that found in G. hirsutum (283 NAC members, Sun et al., 2018), but is about twice as much as that found in G. raimondii (145, Shang et al., 2013) and G. arboreum (141, Shang et al., 2016; Fan et al., 2018). The difference in the size of $N A C$ genes is because the genomes of tetraploid species possess more $N A C$ genes than the diploid species, presumably because an allopolyploidization event occurred in G. babardense and G. hirsutum approximately 1.7-1.9 Mya (Hu et al., 2019). GbNAC genes were unevenly distributed on 26 chromosomes, and a number of GbNAC genes were clustered on the top or bottom of specific chromosomes (Figure 2). These results are similar to those of the two diploid cotton species, G. raimondii, and G. arboreum (Shang et al., 2013; 2016).

According to the multiple sequence alignment, all the GbNAC proteins contain the highly 
360

361

362

363

364

365

366

367

368

369

370

371

372

373

374

375

376

377

378

379

380

381

382

383

384

385

386

conserved NAC domain with 150-160 amino acids, which can be further divided into five distinct subdomains (A-E) at the N-terminal. However, a number of GbNAC proteins have an atypical NAC domain pattern. For example, three GbNAC proteins (GbNAC068, GbNAC200, and GbNAC226) have only conserved subdomain A, while four GbNAC proteins (GbNAC033, GbNAC092, GbNAC109, and GbNAC242) have only subdomain D. In addition, the previously reported GbNAC1 lacks the conserved subdomains B, C, and E (Wang et al., 2016). NAC proteins lacking one to four subdomains were also observed in Arabidopsis, rice, and radish (Ooka et al., 2003; Nuruzzaman et al. 2010; Karanja et al., 2017). Thus, the atypical NAC domain pattern appears to be common among NAC proteins from diverse plant species. Previous studies revealed that subdomains $\mathrm{A}, \mathrm{C}$, and $\mathrm{D}$ have higher levels of conservation than subdomains $\mathrm{B}$ and $\mathrm{E}$, and play important roles in the function of NAC genes (Ooka et al., 2003). Subdomain A has the ability to form a helical structure and is involved in the formation of homodimers or heterodimers with other NAC domain proteins (Jensen et al., 2010). Subdomain D contains the nuclear localization signal and subdomain $\mathrm{C}$ is associated with DNA binding activity (Hernandez \& Sanan-Mishra 2017). In this study, most GbNAC proteins (196/270) contain subdomains A, C, and D, whereas only 48 GbNAC proteins have subdomain B (Figure 5). Furthermore, we found 231 GbNAC proteins contained a conserved histidine residue in subdomain D. A recent study revealed that the conserved histidine residue is present in $80 \%$ of Arabidopsis NAC members and functioned as a switch to regulate both pH-dependent homodimerization and DNA binding (Kang et al., 2019). Thus, the subdomain D may function not only in nuclear localization but also in the formation of functional dimers and DNA-binding.

$N A C$ gene duplication was investigated in sea-island cotton genome. One hundred and fifty-four duplicated $N A C$ gene pairs including 148 segmental duplication pairs and 6 tandem duplication pairs were revealed in sea-island cotton (Figure 3). Therefore, it can be concluded that segmental duplication dominates the expansion of the $N A C$ gene family in the G. barbadense genome. Furthermore, most duplicated gene pairs had undergone strong purifying selection during evolution, indicating that purifying selection played pivotal roles in the confinement of the GbNAC 
gene functions, which was further confirmed by the similar expression pattern of most duplication gene pairs. Previous studies have showed that the A and D ancestor genomes diverged approximately 6.2-7.1 Mya (Hu et al., 2019). In this study, about half of the duplication events occurred after the divergence of the two diploid progenitors (Table S3). Synteny analysis indicated that the collinearity of $N A C$ genes for the four Gossypium species is highly conserved. However, the level of collinearity is different. G. barbadense and G. hirsutum have the highest level of collinearity, while G. barbadense and G. arboretum have the lowest level of collinearity (Table S4). The difference is probably attributed to the genomic characteristics and evolution history of cotton. G. barbadense and G. hirsutum evolved from a common tetraploid ancestor and diverged approximately 0.4-0.6 Mya (Hu et al., 2019), which leads to high collinearity for the two tetraploid species. Nevertheless, the A subgenome of G. barbadense has large chromosome inversions in comparison with $G$. arboretum due to chromosomal rearrangement after allopolyploidization (Wang et al., 2019), which results in relatively low collinearity between G. barbadense and $G$. arboretum.

\section{Differential expression of $G b N A C$ genes in response to Verticillium wilt}

Cotton VW is a destructive soil-borne fungal disease and dramatically reduces the yield and quality of cotton. VW was first reported in Virginia, USA, in 1914 and now occurs worldwide. Over the past century, substantial efforts have been made to develop ways to control VW, and a number of genes, such as Gh_A10G2076,GhATAF1,GbERF1, GbWRKY1, and GbRLK, have been revealed to be associated with VW resistance (Li et al., 2017; He et al., 2016; Guo et al., 2016; Li et al., 2014; Jun et al., 2015). However, whether the NAC genes could play possible role in response to VW in G. barbadense is still in question. In this study, $62 G b N A C$ genes were identified to be significantly up- or down-regulated in roots inoculated with $V$. dahliae at all six time-points (Figure S2 and Table S9). These genes are all from the 10 phylogenetic groups, except group V, and contain at least one cis-regulatory element involved in stress responses (Table S7). We also found that $G b N A C$ genes in each duplicated gene pair had similar expression patterns in response to VW infection. The functional redundancy of duplicated genes may be related to their similar 
414 gene structure, motifs, and cis-regulatory elements. In Arabidopsis, five $N A C$ genes (ANAC016, $415 A N A C 036, A N A C 037, A N A C 061, A N A C 081$, and $A N A C 091$ ) were significantly up-regulated after 416 chitin treatment (Libault et al., 2007). Chitin is an elicitor of plant defense responses and its 417 elicitation plays important role in plant defense to fungal pathogens. Among the $62 G b N A C$ genes, 41810 genes clustered with $A N A C 081$ are from group IV; 13 genes along with $A N A C 036$ and 419 ANAC061 belong to group VI; 4 genes and ANAC091 are from group VII, and 3 genes and 420 ANAC037 are from group I (Table S9).

421 Previously, an upland cotton ATAF subfamily NAC gene, GhATAF1 (DT549350), was reported 422 to be up-regulated by $V$. dahliae inoculation. Cotton plants overexpressing GhATAF1 increased susceptibility to pathogen $V$. dahliae (He et al., 2016). In our study, GbNAC164, an ortholog to GhATAF1, was down-regulated by $V$. dahliae infection. The different expression pattern may be caused by cotton genotypes and/or $V$. dahliae strains. GbNAC164 was investigated in $G$. barbadense cv. 7124 treated with a highly virulent $V$. dahliae strain V991, while GhATAF1 was analyzed in $G$. hirsutum cv. YZ1 treated with a moderately aggressive $V$. dahliae strain ICD3-2. Sea-island cotton $G b N A C 1$ ( $G b N A C 220$ in this study) belongs to the Tobacco elicitor-responsive gene encoding NAC-domain protein (TERN) subgroup and was down-regulated by VW. Cotton plants silencing $G b N A C l$ reduce resistance to $\mathrm{VW}$, whereas transgenic Arabidopsis lines overexpressing GbNACl enhance resistance to VW compared to wild type (Wang et al., 2016). GbNAC220 was also down-regulated by VW in our study. In addition, MeJA and SA are important pathogen-related hormonal regulators. Among the 62 GbNAC genes, 31 and 22 genes contain MeJA- and SA-responsive elements, respectively, and 12 genes contain both MeJA- and SAresponsive elements (Table S7), indicating the expression of these genes may be regulated by MeJA and/or SA. These results were further verified by $\mathrm{qPCR}$ analysis. The qPCR results indicated GbNAC003, GbNAC137, GbNAC140, GbNAC215, and GbNAC248 were sensitive to MeJA and SA treatments (Figure 8), which was in agreement with the results of cis-regulatory element analysis (Table S7). GbNAC164 was up-regulated by MeJA and SA treatment. Moreover, 
441 was consistent with GhATAFl (He et al., 2016). Thus, these findings suggest that up-regulating of 442 the 62 GbNAC genes may result in increased or decreased VW resistance in cotton and these genes 443 can be candidate genes for in-depth study on VW resistance.

444 Interestingly, GhATAF1 was also up-regulated by ABA, cold, and salt treatments. Overexpression 445 of GhATAF1 confers transgenic cotton improved salt tolerance (He et al., 2016). Similarly, 446 Transgenic GbNAC1 results in enhanced drought tolerance in Arabidopsis (Wang et al., 2016). 447 The $N A C$ genes $A N A C 019, A N A C 055$ and $A N A C 072$ from Arabidopsis were up-regulated by 448 drought, salt, and ABA treatments. Transgenic either ANAC019, ANAC055 or ANAC072 conferred 449 Arabidopsis plants enhanced drought tolerance. The finding suggests that the $62 G b N A C$ genes 450 may play role in response to abiotic stresses.

\section{CONCLUSIONS}

452

453

454

In this study, the plant-specific $N A C$ gene family in sea-island cotton was characterized with particular focus on their responses to VW infection. A total of 270 GbNAC genes were identified and characterized in sea-island cotton. The gene structure, chromosomal distribution, gene duplication, conserved motif, cis-elements, and expression profiles of the $G b N A C$ genes were analyzed. Furthermore, expression profile analyses revealed that $62 G b N A C$ genes may play crucial role in response to VW infection. However, further functional data are required to evaluate each $G b N A C$ gene. Overall, our results will provide new insights for plant engineering programs so that economically important traits for cotton can be developed, including improved resistance to VW.

\section{REFERENCES}

Allu AD, Brotman Y, Xue GP, Balazadeh S. 2016. Transcription factor $A N A C 032$ modulates JA/SA signaling in response to Pseudomonas syringae infection. EMBO Reports 17 (11): 15781589.

Artimo P, Jonnalagedda M, Arnold K, Baratin D, Csardi G, de Castro E, Duvaud S, Flegel 
466

467

468

469

470

471

472

473

474

475

476

477

V, Fortier A, Gasteiger E, Grosdidier A, Hernandez C, Ioannidis V, Kuznetsov D, Liechti R, Moretti S, Mostaguir K, Redaschi N, Rossier G, Xenarios I, Stockinger H. 2012. ExPASy: SIB bioinformatics resource portal. Nucleic Acids Research 40 (W1): W597-W603.

Bailey TL, Johnson J, Grant CE, Noble WS. 2015. The MEME Suite. Nucleic Acids Research 43 (W1): W39-W49.

Chen JY, Huang JQ, Li NY, Ma XF, Wang JL, Liu C, Liu YF, Liang Y, Bao YM, Dai XF. 2015. Genome-wide analysis of the gene families of resistance gene analogues in cotton and their response to verticillium wilt. BMC Plant Biology 15: 148.

Dai X, Zhuang Z, Zhao PX. 2018. psRNATarget: a plant small RNA target analysis server (2017 release). Nucleic Acids Research 46 (W1): W49-W54.

Du H, Yang SS, Liang Z, Feng BR, Liu L, Huang YB, Tang YX. 2012. Genome-wide analysis of the MYB transcription factor superfamily in soybean. BMC Plant Biology 12: 106.

Fan K, Bibi N, Gan S, Li F, Yuan S, Ni M, Wang M, Shen H, Wang X. 2015. A novel NAP member GhNAP is involved in leaf senescence in Gossypium hirsutum. Journal of Experimental Botany 66 (15): 4669-4685.

Fan K, Li F, Chen J, Li Z, Lin W, Cai S, Liu J, Lin W. 2018. Asymmetric evolution and expansion of the NAC transcription factor in polyploidized cotton. Frontiers in Plant Science 9: 47.

Fang Y, Xie K, Xiong L. 2014. Conserved miR164-targeted NAC genes negatively regulate drought resistance in rice. Journal of Experimental Botany 65 (8): 2119-2135.

Finn RD,Coggill P, Eberhardt RY, Eddy SR, Mistry J, Mitchell AL, Potter SC, Punta M, Qureshi M, Sangrador-Vegas A, Salazar GA, Tate J, Bateman A. 2016. The Pfam protein families database: towards a more sustainable future. Nucleic Acids Research 44 (Database issue): D279-D285. 
490

491

492

493

494

495

496

497

498

499

500

501

502

503

504

505

506

507

508

509

510

511

512

513

514

Guo W, Jin L, Miao Y, He X, Hu Q, Guo K, Zhu L, Zhang X. 2016. An ethylene responserelated factor, GbERF1-like, from Gossypium barbadense improves resistance to Verticillium dahliae via activating lignin synthesis. Plant Molecular Biology 91 (3): 305-318.

Hernandez Y, Sanan-Mishra N. 2017. miRNA mediated regulation of NAC transcription factors in plant development and environment stress response. Plant Gene 11 (Part B): 190-198.

He X, Zhu L, Xu L, Guo W, Zhang X. 2016. GhATAF1, a NAC transcription factor, confers abiotic and biotic stress responses by regulating phytohormonal signaling networks. Plant Cell Reports 35 (10): 2167-2179.

Higo K, Ugawa Y, Iwamoto M, Korenaga T. 1999. Plant cis-acting regulatory DNA elements (PLACE) database: 1999. Nucleic Acids Research 27 (1): 297-300.

Hu B, Jin J,Guo AY, Zhang H, Luo J, Gao G. 2015. GSDS 2.0: an upgraded gene feature visualization server. Bioinformatics 31 (1): 1296-1297.

Hu H, Dai M, Yao J, Xiao B, Li X, Zhang Q, Xiong L. 2006. Overexpressing a $N A M$, ATAF, and $C U C(N A C)$ transcription factor enhances drought resistance and salt tolerance in rice. Proceedings of the National Academy of Sciences of the United States of America 103 (35): 1298712992.

Hu Y, Chen J, Fang L, Zhang Z, Ma W, Niu Y, Ju L, Deng J, Zhao T, Lian J, Baruch K, Fang D, Liu X, Ruan YL, Rahman MU, Han J, Wang K, Wang Q, Wu H, Mei G, Zang Y, Han Z, Xu C, Shen W, Yang D, Si Z, Dai F, Zou L, Huang F, Bai Y, Brodt A, Ben-Hamo H, Zhu X, Zhou B, Guan X, Zhu S, Chen X, Zhang T. 2019. Gossypium barbadense and Gossypium hirsutum genomes provide insights into the origin and evolution of allotetraploid cotton. Nature Genetics 51 (4): 739-748.

\section{Jensen MK, Kjaersgaard T, Nielsen MM, Galberg P, Petersen K, O'Shea C, Skriver K. 2010.}

The Arabidopsis thaliana NAC transcription factor family: structure-function relationships and determinants of ANAC019 stress signaling. Biochemical Journal 426 (2): 183-196. 
515

516

517

518

519

520

521

522

523

524

525

526

527

528

529

530

531

532

533

534 535

536

537

538

539

Jun Z, Zhang Z, Gao Y, Zhou L, Fang L, Chen X, Ning Z, Chen T, Guo W, Zhang T. 2015.

Overexpression of $G b R L K$, a putative receptor-like kinase gene, improved cotton tolerance to Verticillium wilt. Scientific reports 5: 15048.

Kang M, Kim S, Kim HJ, Shrestha P, Yun JH, Phee BK, Lee W, Nam HG, Chang I. 2018. The C-domain of the NAC transcription factor ANAC019 is necessary for pH-tuned DNA binding through a histidine switch in the N-domain. Cell Reports 22 (5): 1141-1150.

Karanja BK, Xu L, Wang Y, Muleke EM, Jabir BM, Xie Y, Zhu X, Cheng W, Liu L. 2017. Genome-wide characterization and expression profiling of NAC transcription factor genes under abiotic stresses in radish (Raphanus sativus L.) PeerJ 5: e4172.

Kim SG, Lee S, Seo PJ, Kim SK, Kim JK, Park CM. 2010. Genome-scale screening and molecular characterization of membrane-bound transcription factors in Arabidopsis and rice. Genomics 95 (1): 56-65.

Krogh A, Larsson B, von Heijne G, Sonnhammer EL. 2001. Predicting transmembrane protein topology with a hidden markov model: application to complete genomes. Journal of Molecular Biology 305 (3): 567-580.

Krzywinski M, Schein JE, Birol I, Connors J, Gascoyne R, Horsman D, Jones SJ, Marra MA. 2009. Circos: an information aesthetic for comparative genomics. Genome Research 19 (9): $1639-1645$.

Kumar S, Stecher G, Li M, Knyaz C, Tamura K. 2018. MEGA X: molecular evolutionary genetics analysis across computing platforms. Molecular Biology and Evolution 35 (6): 15471549.

Larkin MA, Blackshields G, Brown NP, Chenna R, McGettigan PA, McWilliam H, Valentin F, Wallace IM, Wilm A, Lopez R, Thompson JD, Gibson TJ, Higgins DG. 2007. Clustal W and Clustal X version 2.0. Bioinformatics 23 (21): 2947-2948.

Lescot M, Dehais P, Thijs G, Marchal K, Moreau Y, Van de Peer Y, Rouze P, Rombauts S. 
540

541

542

543

544

545

546

547

548

549

550

551

552

553

554

555

556

557

558

559

560

561

562

563

2002. PlantCARE, a database of plant cis-acting regulatory elements and a portal to tools for in silico analysis of promoter sequences. Nucleic Acids Research 30 (1): 325-327.

Letunic I, Bork P. 2018. 20 years of the SMART protein domain annotation resource. Nucleic Acids Research 46 (D1): D493-D496.

Lex A, Gehlenborg N, Strobelt H, Vuillemont R, Pfister H. 2014. UpSet: visualization of intersecting sets. IEEE Transactions on Visualization and Computer Graphics 20 (12): 1983-1992.

Li C, He X, Luo X, Xu L, Liu L, Min L, Jin L, Zhu L, Zhang X. 2014. Cotton WRKY1 mediates the plant defense-to-development transition during infection of cotton by Verticillium dahliae by activating JASMONATE ZIM-DOMAIN1 expression. Plant Physiology 166 (4): 2179-2194.

Li S, Wang N, Ji D, Xue Z, Yu Y, Jiang Y, Liu J, Liu Z, Xiang F. 2016. Evolutionary and functional analysis of membrane-bound NAC transcription factor genes in soybean. Plant Physiology 172 (3): 1804-1820.

Li T, Ma X, Li N, Zhou L, Liu Z, Han H, Gui Y, Bao Y, Chen J, Dai X. 2017. Genome-wide association study discovered candidate genes of Verticillium wilt resistance in upland cotton (Gossypium hirsutum L.). Plant Biotechnology Journal 15 (12): 1520-1532.

Li W, Zhou Z, Meng Y, Xu N, Fok M. 2009. Modeling boll maturation period, seed growth, protein, and oil content of cotton (Gossypium hirsutum L.) in China. Field Crops Research 112 (2-3): 131-140.

Libault M, Wan J, Czechowski T, Udvardi M, Stacey G. 2007. Identification of 118 Arabidopsis transcription factor and 30 ubiquitin-ligase genes responding to chitin, a plant-defense elicitor. Molecular Plant-Microbe Interactions 20 (8): 900-911.

Livak KJ, Schmittgen TD. 2001. Analysis of relative gene expression data using real-time quantitative PCR and the 2- $-\Delta \mathrm{CT}$ method. Methods 25 (4): 402-408.

Liu M, Ma Z, Sun W, Huang L, Wu Q, Tang Z, Bu T, Li C, Chen H. 2019. Genome-wide 
564

565

566

567

568

569

570

571

572

573

574

575

576

577

578

579

580

581

582

583

584

585

586

587

588

analysis of the NAC transcription factor family in tartary buckwheat (Fagopyrum tataricum). BMC Genomics 20: 113.

Liu X, Wang T, Bartholomew E, Black K, Dong M, Zhang Y, Yang S, Cai Y, Xue S, Weng Y, Ren H. 2018. Comprehensive analysis of NAC transcription factors and their expression during fruit spine development in cucumber (Cucumis sativus L.) Horticulture Research 5: 31.

Liu X, Zhao B, Zheng HJ, Hu Y, Lu G, Yang CQ, Chen JD, Chen JJ, Chen DY, Zhang L, Zhou Y, Wang LJ, Guo WZ, Bai YL, Ruan JX, Shangguan XX, Mao YB, Shan CM, Jiang JP, Zhu YQ, Jin L, Kang H, Chen ST, He XL, Wang R, Wang YZ, Chen J, Wang LJ, Yu ST, Wang BY, Wei J, Song SC, Lu XY, Gao ZC, Gu WY, Deng X, Ma D, Wang S, Liang WH, Fang L, Cai CP, Zhu XF, Zhou BL, Jeffrey Chen Z, Xu SH, Zhang YG, Wang SY, Zhang TZ, Zhao GP, Chen XY. 2015. Gossypium barbadense genome sequence provides insight into the evolution of extra-long staple fiber and specialized metabolites. Scientific reports 5: 14139.

Metsalu T, Vilo J. 2015. ClustVis: a web tool for visualizing clustering of multivariate data using principal component analysis and heatmap. Nucleic Acids Research 43 (W1): W566-W570.

Nuruzzaman M, Manimekalai R, Sharoni AM, Satoh K, Kondoh H, Ooka H, Kikuchi S. 2010. Genome-wide analysis of NAC transcription factor family in rice. Gene 465 (1-2): 30-44.

Nuruzzaman M, Sharoni AM, Kikuchi S. 2013. Roles of NAC transcription factors in the regulation of biotic and abiotic stress responses in plants. Frontiers in Microbiology 4: 248.

Olsen AN, Ernst HA, Leggio LL, Skriver K. 2005. NAC transcription factors: structurally distinct, functionally diverse. Trends in Plant Science 10 (2): 79-87.

Shang H, Li W, Zou C, Yuan Y. 2013. Analysis of the NAC transcription factor gene family in Gossypium raimondii Ulbr.: chromosomal location, structure, phylogeny, and expression patterns. Journal of Integrative Plant Biology 55 (7): 663-676.

Shang H, Wang Z, Zou C, Zhang Z, Li W, Li J, Shi Y, Gong W, Chen T, Liu A, Gong J, Ge Q, Yuan Y. 2016. Comprehensive analysis of NAC transcription factors in diploid Gossypium: 
589

590

591

592

593

594

595

596

597

598

599

600

601

602

603

604

605

606

607

608

609

610

611

612

613

sequence conservation and expression analysis uncover their roles during fiber development. Science China Life Sciences 59 (2): 142-153.

Souer E, van Houwelingen A, Kloos D, Mol J, Koes R. 1996. The no apical meristem gene of Petunia is required for pattern formation in embryos and flowers and is expressed at meristem and primordia boundaries. Cell 85 (2): 159-170.

Sun H, Hu M, Li J, Chen L, Li M, Zhang S, Zhang X, Yang X. 2018. Comprehensive analysis of NAC transcription factors uncovers their roles during fiber development and stress response in cotton. BMC Plant Biology 18: 150.

Sun Q, Jiang H, Zhu X, Wang W, He X, Shi Y, Yuan Y, Du X, Cai Y. 2013. Analysis of seaisland cotton and upland cotton in response to Verticillium dahliae infection by RNA sequencing. BMC Genomics 14: 852 .

Takasaki H, Maruyama K, Takahashi F, Fujita M, Yoshida T, Nakashima K, Myouga F, Toyooka K, Yamaguchi-Shinozaki K, Shinozaki K. 2015. SNAC-As, stress-responsive NAC transcription factors, mediate ABA-inducible leaf senescence. Plant Journal 84 (6): 1114-1123.

Vatansever R, Koc I, Ozyigit II, Sen U, Uras ME, Anjum NA, Pereira E, Filiz E. 2016. Genome-wide identification and expression analysis of sulfate transporter (SULTR) genes in potato (Solanum tuberosum L.). Planta 244 (6): 1167-1183.

Voorrips R. 2002. MapChart: software for the graphical presentation of linkage maps and QTLs. Journal of Heredity 93 (1):77-78.

Wang Y, Tang H, DeBarry JD, Tan X, Li J, Wang X, Lee TH, Jin H, Marler B, Guo H, Kissinger JC, Paterson AH. 2012. MCScanX: a toolkit for detection and evolutionary analysis of gene synteny and collinearity. Nucleic Acids Research 40 (7): e49.

Wang M, Tu L, Yuan D, Zhu D, Shen C, Li J, Liu F, Pei L, Wang P, Zhao G, Ye Z, Huang H, Yan F, Ma Y, Zhang L, Liu M, You J, Yang Y, Liu Z, Huang F, Li B, Qiu P, Zhang Q, Zhu L, Jin S, Yang X, Min L, Li G, Chen LL, Zheng H, Lindsey K, Lin Z, Udall JA, Zhang 
614 X. 2019. Reference genome sequences of two cultivated allotetraploid cottons, Gossypium 615 hirsutum and Gossypium barbadense. Nature Genetics 51 (2): 224-229.

616 Wang W, Yuan Y, Yang C, Geng S, Sun Q, Long L, Cai C, Chu Z, Liu X, Wang G, Du X, 617 Miao C, Zhang X, Cai Y. 2016. Characterization, expression, and functional analysis of a novel 618 NAC gene associated with resistance to Verticillium wilt and abiotic stress in cotton. G3: Genes, 619 Genomes, Genetics 6 (12): 3951-3961.

620

621

622

Yokotani N, Tsuchida-Mayama T, Ichikawa H, Mitsuda N, Ohme-Takagi M, Kaku H, Minami E, Nishizawa Y. 2014. OsNAC111, a blast disease-responsive transcription factor in rice, positively regulates the expression of defense-related genes. Molecular Plant-Microbe Interactions 27 (10): 1027-1034.

Yu CS, Chen YC, Lu CH, Hwang JK. 2006. Prediction of protein subcellular localization. Proteins: Structure, Function and Bioinformatics 64 (3): 643-651.

Zhao F, Ma J, Li L, Fan S, Guo Y, Song M, Wei H, Pang C, Yu S. 2016. GhNAC12, a neutral candidate gene, leads to early aging in cotton (Gossypium hirsutum L). Gene 576 (1 Pt 2): 268274.

Zhang J, Huang GQ, Zou D, Yan JQ, Li Y, Hu S, Li XB. 2018. The cotton (Gossypium hirsutum) NAC transcription factor (FSN1) as a positive regulator participates in controlling secondary cell wall biosynthesis and modification of fibers. New Phytologist 217 (2): 625-640.

Zhang J, Yu J, Pei W, Li X, Said J, Song M, Sanogo S. 2015. Genetic analysis of Verticillium wilt resistance in a backcross inbred line population and a meta-analysis of quantitative trait loci for disease resistance in cotton. BMC Genomics 16: 577.

Zhang Y, Wang XF, Ding ZG, Ma Q, Zhang GR, Zhang SL, Li ZK, Wu Q, Zhang GY, Ma ZY. 2013. Transcriptome profiling of Gossypium barbadense inoculated with Verticillium dahliae provides a resource for cotton improvement. BMC Genomics 14: 637.

Zhang Z, Li J, Zhao XQ, Wang J, Wong GK, Yu J. 2006. KaKs_Calculator: calculating Ka and 
639 Ks through model selection and model averaging. Genomics Proteomics Bioinformatics 4 (4): $640 \quad 259-263$. 


\section{Figure 1}

Phylogenetic tree of the $270 \mathrm{GbNAC}$ proteins.

Multiple sequence alignment of NAC domain sequences of $G$. barbadense and Arabidopsis was performed using ClustalW. MEGA X was used to construct the neighbor-joining (NJ) tree with 1000 bootstrap replicates. Various colors indicate different groups of GbNACs. 


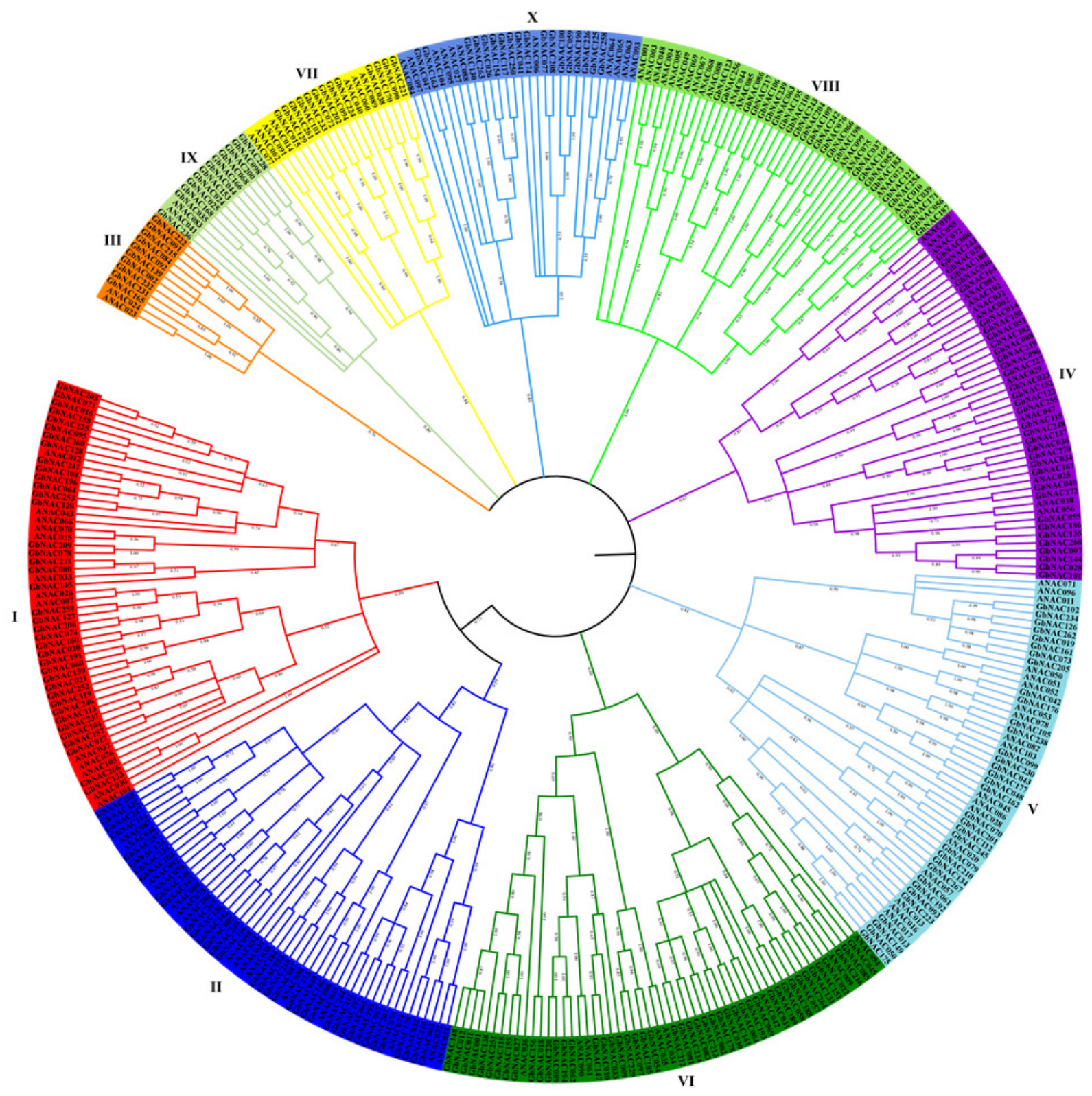


Figure 2

Chromosomal locations of the GbNAC genes.

Grey bars denote the $G$. babardense chromosome. Scale bar on the left indicates the chromosome lengths $(\mathrm{Mb})$. The letter $\mathrm{A}-\mathrm{M}$ indicates the $\mathrm{A}$-subgenome chromosome $\mathrm{A} 01-\mathrm{A} 13$, and $\mathrm{N}-\mathrm{O}$ indicates the $\mathrm{D}$-subgenome chromosome D01-D13, respectively. The vertical red lines on the left of each chromosome indicate tandem duplicated genes.

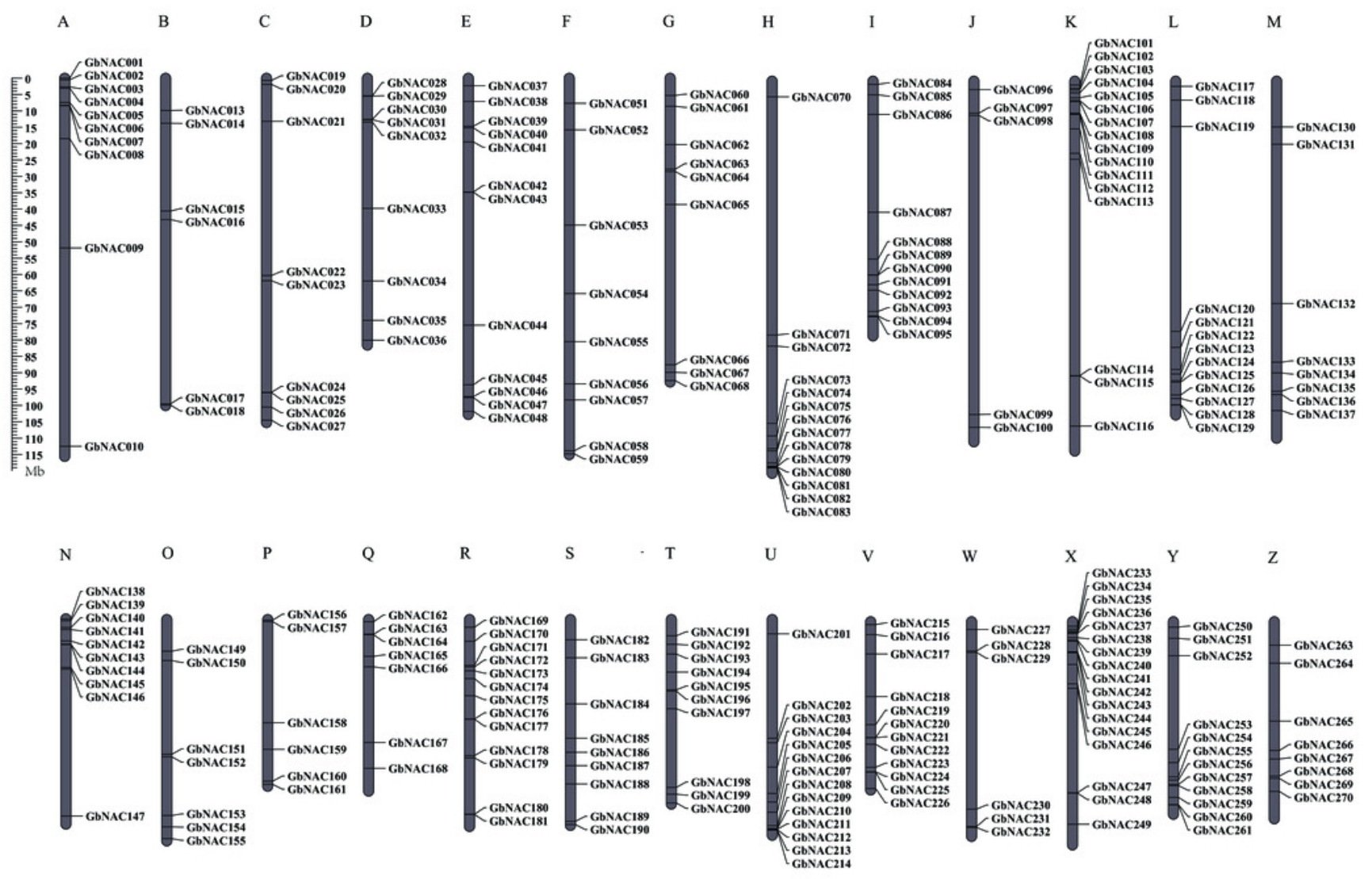


Figure 3

Circos diagram of the GbNAC duplication pairs in G. barbadense.

143 GbNAC duplication pairs are linked with green lines. Scale bar marked on the chromosome indicating chromosome lengths (Mb). 


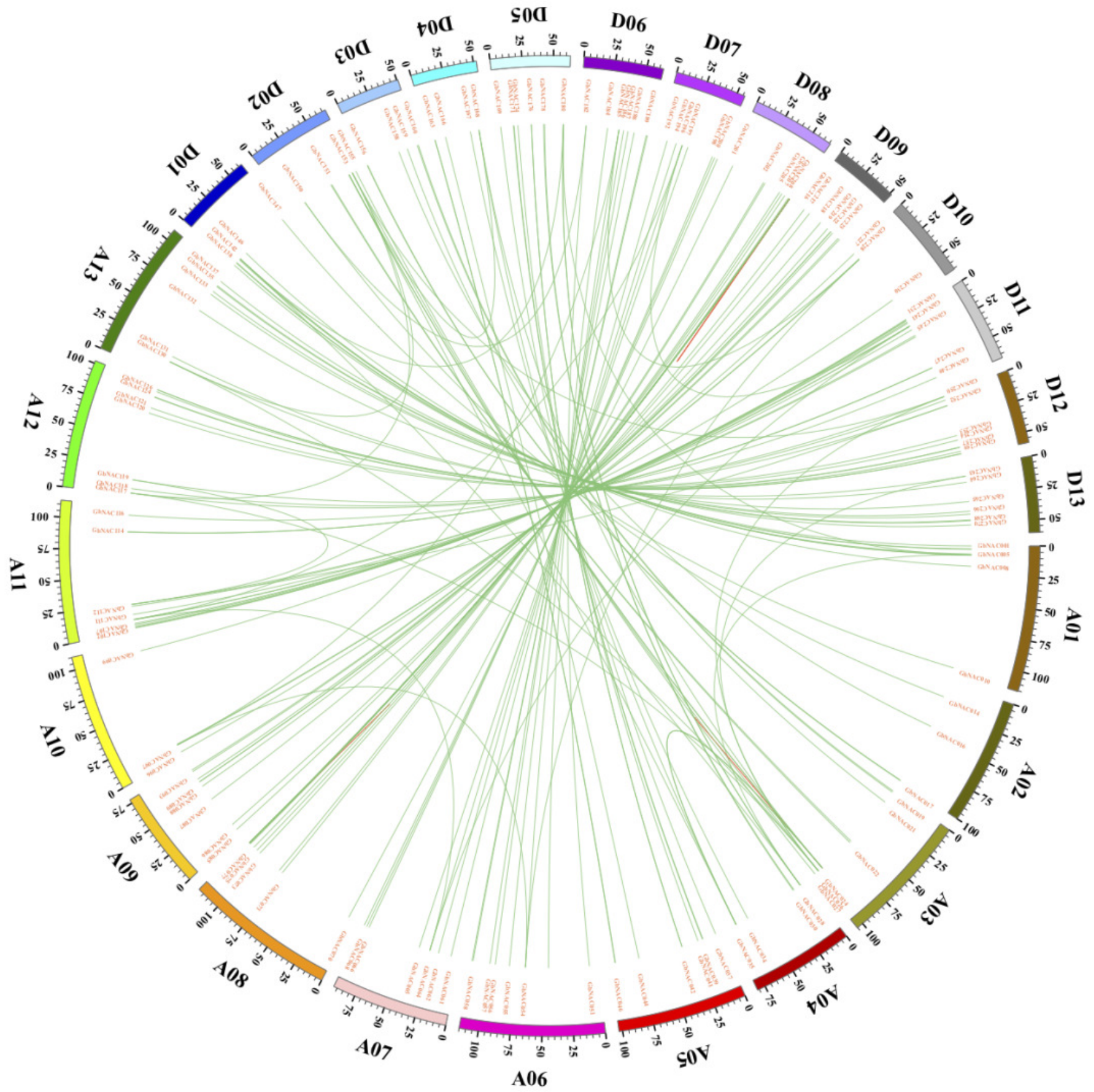




\section{Figure 4}

Synteny analysis of NAC genes between Gossypium barbadense and other plant species.

At, Ga, Gr, Gb, and Gh indicate Arabidopsis thaliana, G. arboreum, G. raimondii, G. barbadense, and G. hirsutum, respectively. A: Collinearity between At and Gb; B: Collinearity between $\mathrm{Ga}$ and $\mathrm{Gb}$; C: Collinearity between $\mathrm{Gr}$ and $\mathrm{Gb}$; D: Collinearity between $\mathrm{Gh}$ and $\mathrm{Gb}$. Gray lines in the background represent the collinear blocks within the genomes of $G$. barbadense and other plant species, while the red lines show the collinear NAC gene pairs. 


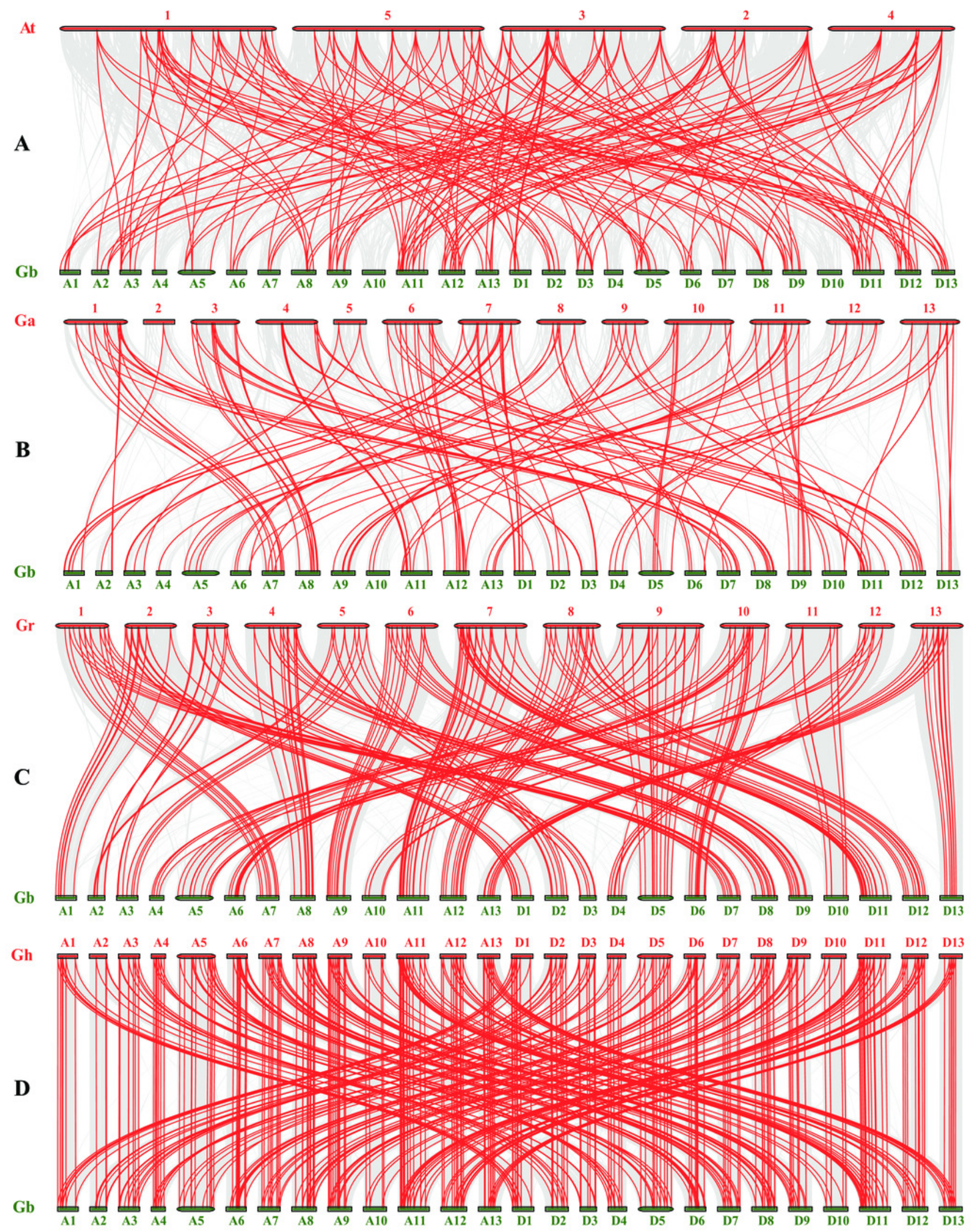




\section{Figure 5}

Putative conserved motifs and gene structures of the GbNAC genes.

A: phylogenetic tree. Multiple sequence alignment of NAC domain sequences of $G$.

barbadense was performed using ClustalW. The neighbor-joining (NJ) tree was constructed using MEGA X with 1000 bootstrap replicates. B: conserved motif. MEME analysis revealed the conserved motifs of the GbNAC proteins. The colored boxes on the right denote 20 motifs. C: gene structure. The yellow boxes, black lines, and green boxes represent exon, intron, and UTR (untranslated region), respectively. 


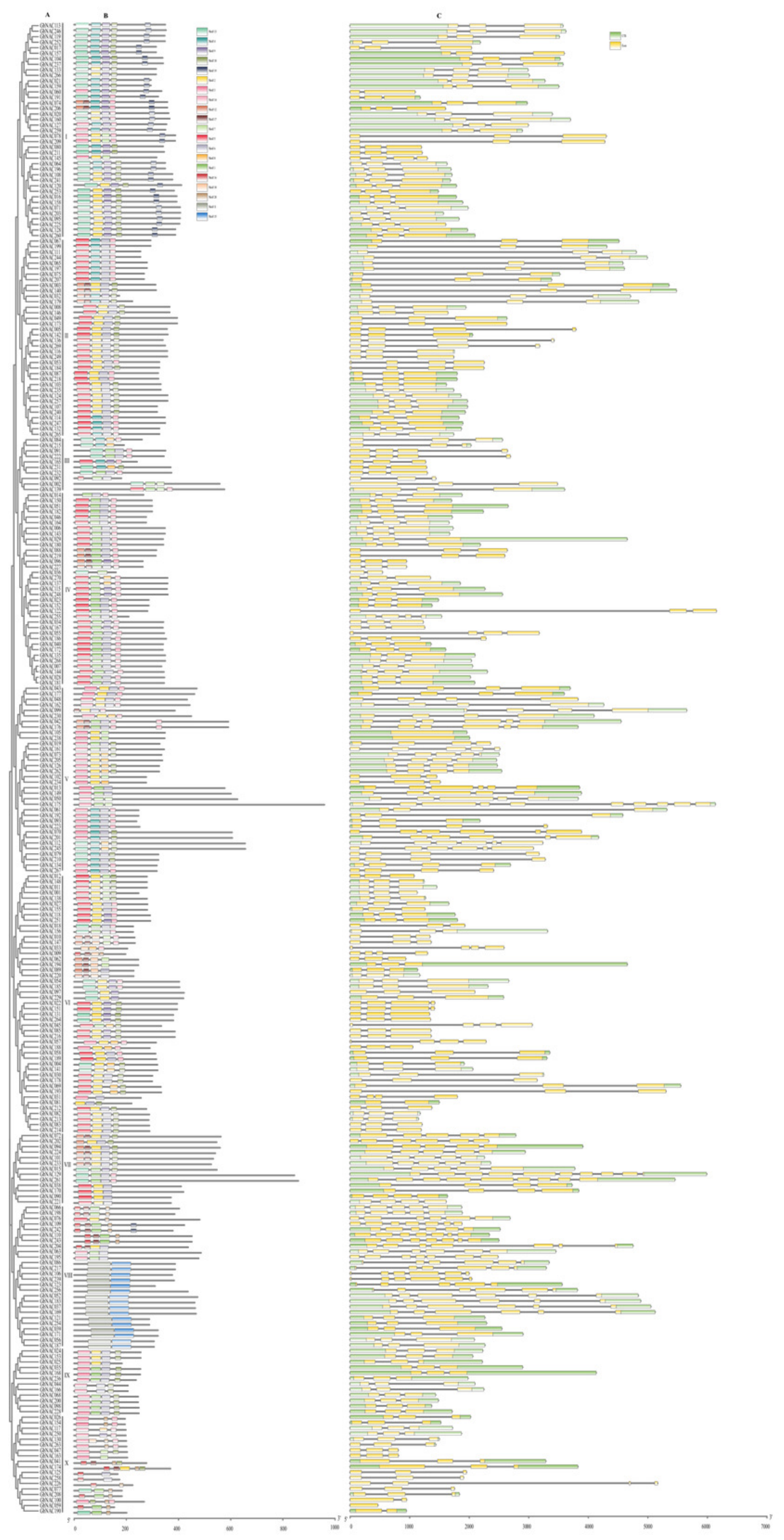

Peer) reviewing PDF | (2019:04:36412:3:0:NEW 26 Sep 2019) 
Figure 6

Conserved subdomains in the NAC domain at the N-terminal of GbNAC proteins

A-E indicate five conserved subdomain A-E, respectively.

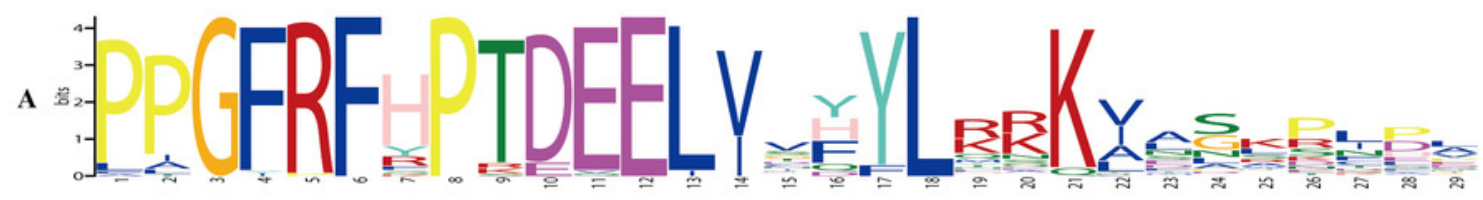

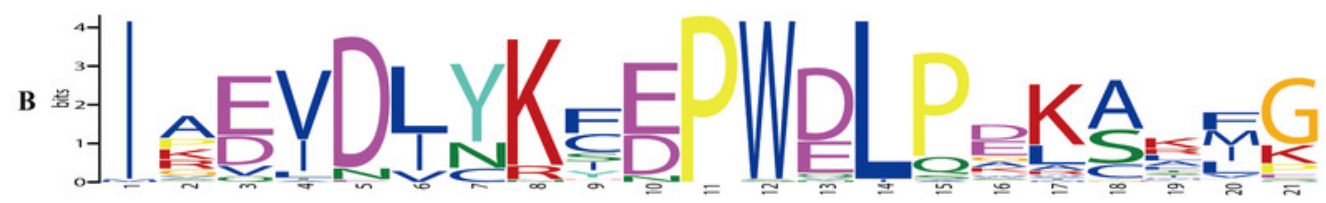

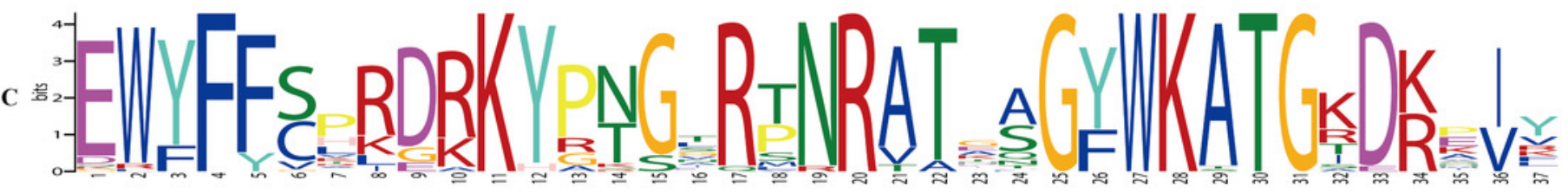

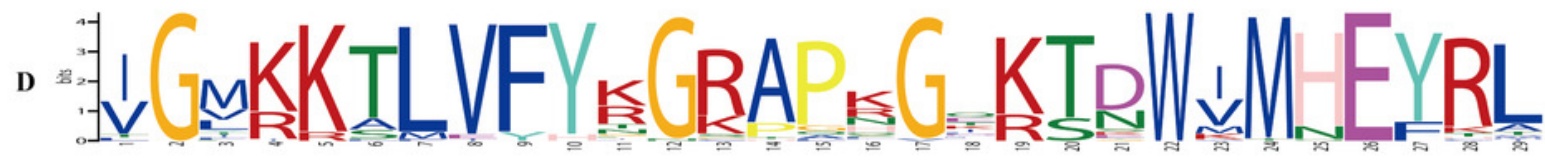

- A 


\section{Figure 7}

Expression profiles of GbNAC genes in roots under Verticillium wilt infection.

The heat map was generated by ClustVis software. The expression data were gene-wise normalized and hierarchically clustered with average linkage. The bar on the right of the heat map indicates relative expression values. Values 2, 0, -2 represent high, intermediated, and low expression, respectively.

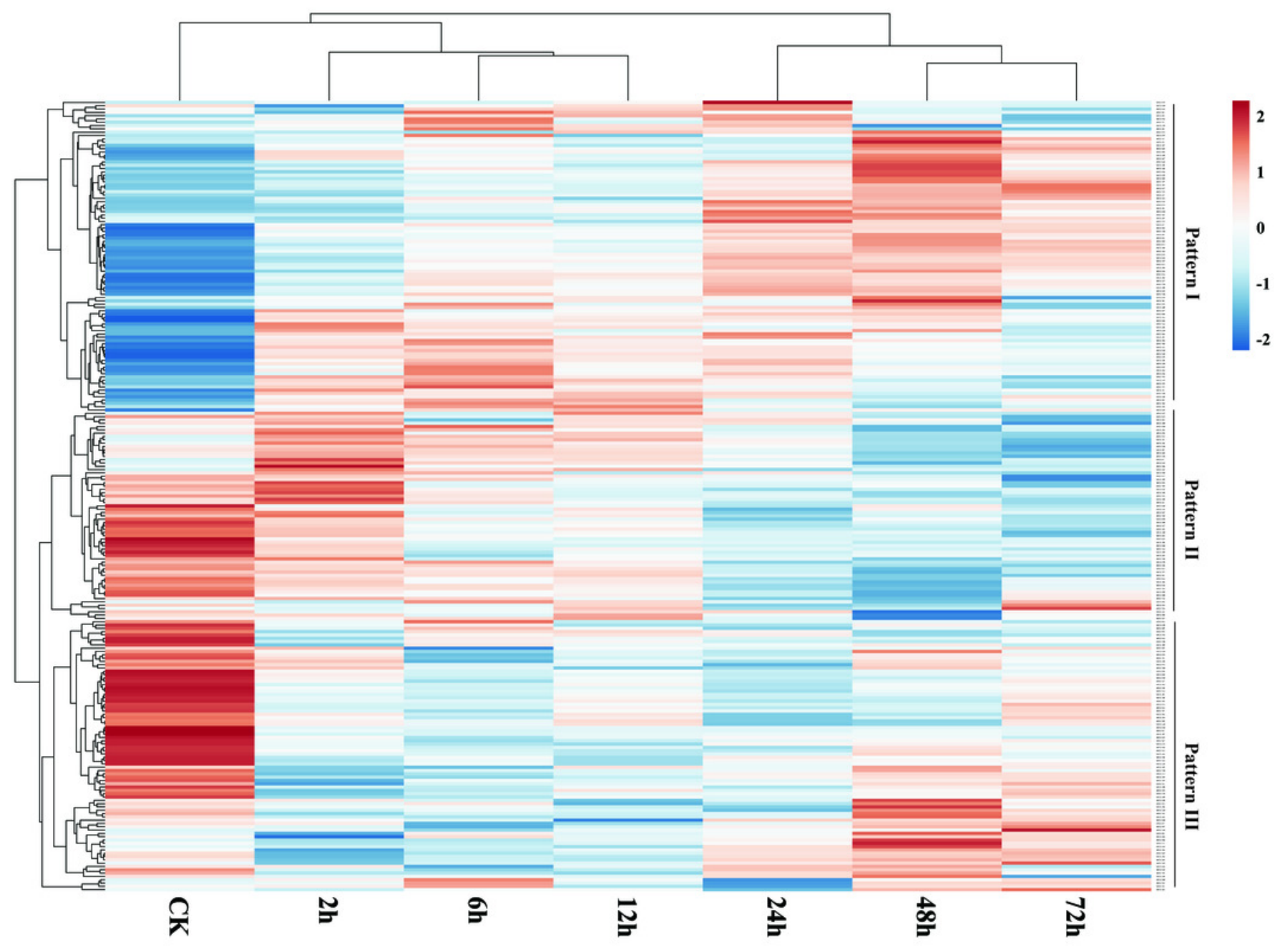


Figure 8

Expression profiles of GbNAC genes in response to hormone MeJA and SA.

qPCR was used to analyze the expression profiles of 15 GbNAC genes under hormone MeJA and SA treatment. The letter A-O indicates the 15 GbNAC genes tested, respectively. The relative expression levels are normalized to GbActin. The data represent the mean of three biological replicates. Significant differences were indicated as * (Tukey's HSD, $P<0.05)$ and ** $(P<0.01)$ above the bars between treatment and control.
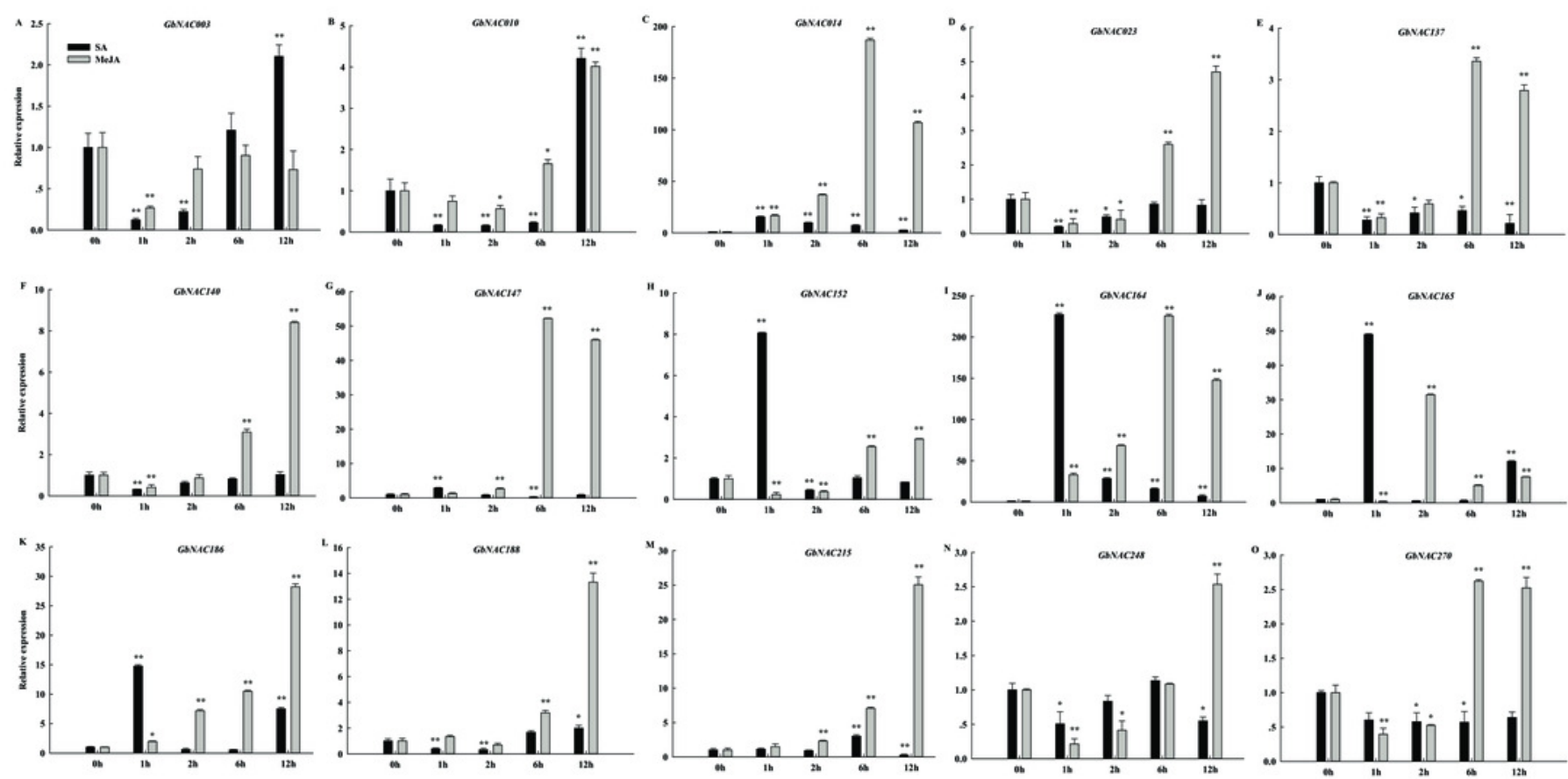